\title{
Significance of endometrial thickness change after human chorionic gonadotrophin triggering in modified natural cycles for frozen-thawed embryo transfer
}

\author{
Jialyu Huang" ${ }^{\#}$, Jiaying Lin ${ }^{1 \#}$, Renfei Cai ${ }^{1}$, Xuefeng Lu ${ }^{1}$, Ning Song ${ }^{2}$, Hongyuan Gao ${ }^{1}$, Yanping Kuang ${ }^{1}$ \\ ${ }^{1}$ Department of Assisted Reproduction, Shanghai Ninth People's Hospital, Shanghai Jiao Tong University School of Medicine, Shanghai, China; \\ ${ }^{2}$ Department of Histology, Embryology, Genetics and Developmental Biology, Shanghai Key Laboratory of Reproductive Medicine, Shanghai Jiao \\ Tong University School of Medicine, Shanghai, China \\ Contributions: (I) Conception and design: J Huang, J Lin, Y Kuang; (II) Administrative support: J Huang, J Lin; (III) Provision of study materials or \\ patients: J Lin, R Cai, X Lu, H Gao, Y Kuang; (IV) Collection and assembly of data: J Huang, J Lin; (V) Data analysis and interpretation: J Huang, J \\ Lin, N Song, Y Kuang; (VI) Manuscript writing: All authors; (VII) Final approval of manuscript: All authors. \\ \#These authors contributed equally to this work. \\ Correspondence to: Yanping Kuang. Department of Assisted Reproduction, Shanghai Ninth People's Hospital, Shanghai Jiao Tong University School \\ of Medicine, 639 Zhizaoju Rd., Shanghai 200011, China. Email: kuangyp9hospital@126.com.
}

Background: Peak endometrial thickness (EMT), measured on the end of follicular phase or early luteal phase, is the most widely used marker for endometrial receptivity during infertility treatment. However, the clinical significance of follicular-to-luteal EMT change remains unclear. We aimed to study whether the change of EMT between the day of human chorionic gonadotrophin (hCG) triggering and the day of frozen-thawed embryo transfer (FET) has any influence on pregnancy outcomes in modified natural cycles (mNCs).

Methods: This was a retrospective cohort study of 2,768 regular ovulatory women who underwent their first mNC-FET cycles from January 2011 to June 2015. Patients were divided into three groups according to the percentage change of EMT from the hCG triggering day to the FET day: $>5 \%$ decrease $(\mathrm{n}=405), \pm 5 \%$ plateau $(n=1,259)$ and $>5 \%$ increase $(n=1,104)$. The main outcome measure was live birth rate.

Results: Live birth rates were 41.9\%, 39.8\% [crude odds ratio (cOR) 0.91, 95\% CI, 0.73-1.15) and 42.4\% (cOR 1.02, 95\% CI, 0.87-1.20) in the EMT plateau, decrease and increase groups, respectively $(\mathrm{P}=0.649)$. Multiple regression analysis did not alter the finding after controlling for a variety of confounders. Compared with the post-trigger EMT plateau group, the adjusted OR of live birth was 0.88 (95\% CI, 0.69-1.12) in the decrease group and 1.05 (95\% CI, 0.88-1.25) in the increase group. Similarly, no significant associations were observed before or after adjustment between EMT change and other pregnancy outcomes including positive hCG test, clinical pregnancy, early miscarriage and ongoing pregnancy.

Conclusions: EMT change from hCG triggering to embryo transfer was not associated with pregnancy chances in mNC-FET cycles. This reassuring finding should provide guidance for physicians and patients when confronted with EMT decrease on the transfer day.

Keywords: Endometrial thickness (EMT); modified natural cycle (NC); frozen-thawed embryo transfer (FET); pregnancy

Submitted Feb 09, 2020. Accepted for publication Sep 25, 2020.

doi: $10.21037 /$ atm-20-1459

View this article at: http://dx.doi.org/10.21037/atm-20-1459 


\section{Introduction}

The utilization of frozen-thawed embryo transfer (FET) has been on a constant rise since the first baby was born of this technique in 1983 (1-3). Besides lowering the risk of ovarian hyperstimulation syndrome, FET has also shown its advantages in improving pregnancy rates and perinatal outcomes by mitigating the detrimental effects of controlled ovarian stimulation (COS) on uterine environment in fresh transfers (4-7). Maximization of FET success depends greatly on the embryo-endometrial synchronization, yet no consensus has been reached on the optimal endometrial preparation regimen among natural cycle $(\mathrm{NC})$, artificial cycle and ovulation induction cycle $(8,9)$.

Assessment of endometrial thickness (EMT), the most widely and conventionally used marker for endometrial receptivity, is routinely performed through transvaginal ultrasonography (TVU) during infertility treatment $(10,11)$. In a number of previous observational studies, patients with thin endometria have been found to have a lower chance of achieving clinical pregnancy $(10,11)$. Among most studies, the EMT was measured solely on the day of human chorionic gonadotrophin (hCG) administration in fresh transfers or the day of progesterone $(\mathrm{P})$ initiation in FET cycles, while other investigators assessed it on the embryo transfer day. In modified NCs (mNCs), a single measurement of EMT on the triggering day was also frequently adopted (12).

However, the thickness of endometrium varies at different time-points during the menstrual cycle (13-21). In the follicular phase, elevated level of estradiol $\left(\mathrm{E}_{2}\right)$ induces proliferation of the functional layer, leading to an increase of EMT and a hypo-echogenic triple-line pattern ideally. However, in the luteal phase, exposure of endometrium to $\mathrm{P}$ produced by corpus luteum can promote the secretory activity in the glands, vascularization of the stroma and pre-decidualization, resulting in a more hyper-echogenic appearance with EMT change (22). Although concerns are frequently voiced by patients with decreased EMT between the end of follicular/estrogen phase and the day of FET, only few studies have explored this issue with controversial results (23-25). These studies are hampered by small sample size, lack of live birth outcome and more importantly, a limited evaluation of EMT change due to their use of artificial cycles for endometrial preparation.

Characterized by a normal process of follicular development and ovulation, modified $\mathrm{NC}(\mathrm{mNC})$ provides a valuable model for investigation in a virtually noninterventional state. The aim of the present study is to determine whether the change of EMT between the day of hCG administration and embryo transfer has any influence on pregnancy outcomes in mNC-FET cycles. We present the following article in accordance with the MDAR checklist and STROBE reporting checklist (available at http://dx.doi.org/10.21037/atm-20-1459).

\section{Methods}

\section{Study design and participants}

The study was conducted in accordance with the Declaration of Helsinki (as revised in 2013). This retrospective cohort study was performed at the Department of Assisted Reproduction of Shanghai Ninth People's Hospital affiliated with Shanghai Jiao Tong University School of Medicine. The Ethics Committee (Institutional Review Board) of the hospital approved our study protocol (No. 2017-211). Individual consent for this retrospective analysis was waived. We included infertile women with regular ovulatory cycles who underwent their first FETs using the mNC protocol from January 2011 to June 2015. Patients after June 2015 were excluded as most EMT data on the transfer day were not recorded in our electronic database. Other exclusion criteria were: (I) history of recurrent miscarriage, defined as three or more spontaneous pregnancy losses; (II) congenital uterine malformations such as uterus unicornis, septate uterus, duplex uterus and uterus bicomis; (III) acquired uterine diseases including intrauterine adhesion, endometrial polyps, submucosal myomas and adenomyosis; and (IV) incomplete records in our electronic database. None of these patients used donor sperm for insemination and the interval from ovum pickup to FET was limited within 1 year. In total, 2,768 patients were ultimately included for further analysis.

\section{Ovarian stimulation and laboratory protocols}

As previously described (26-28), patients were assigned to one of the following four COS regimens with a nonelective freeze-all strategy: gonadotropin-releasing hormone $(\mathrm{GnRH})$ antagonist, GnRH agonist short, mild stimulation and progestin-primed ovarian stimulation (PPOS). Follicular aspiration was scheduled at 34-36 hours after triggering for final oocyte maturation. The retrieved oocytes were fertilized by in vitro fertilization (IVF) and/or intracytoplasmic sperm injection (ICSI) according to semen quality. The zygotes were transferred and sequentially cultured in Early Cleavage Medium (Irvine Scientific, USA) 
and Multiblast Medium (Irvine Scientific, USA) before 2013, while Continuous Single Culture (Irvine Scientific, USA) was changed for the entire embryo developmental stage after 2013. Cleavage-stage embryos were scored on day 3 (D3) based on the Cummins's criteria (29), of which grade I and II embryos were selected for vitrification. Suboptimal D3 embryos (grades III and IV) were extended in culture period and morphologically good blastocysts (grade $\geq 3 \mathrm{BC}$ ) were then vitrified on D5 or D6 according to the Gardner and Schoolcraft's criteria (30). The details of vitrification and thawing procedures have been presented elsewhere (26).

\section{Endometrial preparation and thickness measurement}

Patients undergoing mNC-FET attended for follicular monitoring via serial TVU and blood sampling from D10 of their menstrual cycle. Once the diameter of the leading follicle was $\geq 17 \mathrm{~mm}$ with EMT $\geq 7 \mathrm{~mm}, \mathrm{E}_{2}>150 \mathrm{pg} / \mathrm{mL}$ and $\mathrm{P}<1$ ng/mL, 5,000 IU hCG (Lizhu Pharmaceutical Trading Co., China) was injected for ovulation triggering. The hCG administration was scheduled in the same afternoon in occurrence of spontaneous luteinizing hormone $(\mathrm{LH})$ surge ( $\geq 20 \mathrm{IU} / \mathrm{L}$ ), or at night (9:00 p.m.) if $\mathrm{LH}<20 \mathrm{IU} / \mathrm{L}$. Exogeneous $\mathrm{P}$ intake $(20 \mathrm{mg}$ dydrogesterone twice daily; Duphaston, Abbott Biologicals, USA) was initiated after the presumed day of ovulation, followed by transfer of cleavagestage embryos 3 days later or blastocysts 5 days later. For each patient in one FET cycle, up to two embryos were transferred via the guidance of transabdominal ultrasound. When a pregnancy was achieved, luteal phase support was given until 10 weeks of gestation. The scheme of embryo transfer timing for $\mathrm{mNC}$ preparation is shown in Figure S1A.

Measurement of EMT was conducted by highly experienced sonographers using a Voluson E8 machine (GE Healthcare, Austria) and a $6-\mathrm{MHz}$ transvaginal probe. We considered EMT as the maximal distance from the anterior to the posterior stratum basalis-myometrial junction in the midsagittal plane. On the day of hCG triggering, EMT was routinely recorded during the study period. To further confirm the FET plan and patients' compliance with $\mathrm{P}$ supplementation, we additionally assessed the endometrium on the day of embryo transfer. Cycles were not cancelled in cases of EMT decrease below $7 \mathrm{~mm}$ on the transfer day.

\section{Outcome parameters}

The primary outcome of the study was live birth. Other outcome measures included positive hCG test, clinical pregnancy, intrauterine implantation, early miscarriage and ongoing pregnancy.

Live birth was identified as the delivery of a viable infant at $\geq 24$ weeks of gestation. We defined positive hCG test as increased serum $\beta$-hCG levels ( $\geq 5 \mathrm{IU} / \mathrm{L}$ ) at 14 days after FET. Clinical pregnancy was defined as the visualization of at least one gestational sac on TVU with or without fetal cardiac activity at the gestational age of 7 weeks. The rate of intrauterine implantation was calculated as the ratio of intrauterine gestational sac number over the number of embryos transferred. We defined early miscarriage as spontaneous pregnancy loss before 12 weeks' gestation, while ongoing pregnancy was confirmed as a viable pregnancy beyond 12 weeks of gestation.

\section{Statistical analysis}

Patients were divided into three groups according to the percentage change of EMT from the day of hCG triggering to the FET day: $>5 \%$ decrease, $\pm 5 \%$ plateau and $>5 \%$ increase. This classification is based on the previous study finding that endometrial compaction by more than $5 \%$ could lead to a significant elevation of ongoing pregnancy rate for approximately $20 \%$ in artificial FET cycles (25). The normality of continuous variables was assessed using the Q-Q plots as well as the Shapiro-Wilk and Kolmogorov-Smirnov tests. Normally distributed data were compared by one-way analysis of variance, while KruskalWallis test was applied for data that were not normally distributed. Comparison of categorical variables among the three groups was performed with the use of Chi-square test or Fisher's exact test, as appropriate.

Univariable and multiple logistic regression analyses were employed to evaluate the independent association between EMT change and pregnancy outcomes. The following potential confounders were entered for adjusted analyses: maternal age $(<30,30-34,35-37,38-40$ or $\geq 41$ years), maternal body mass index (BMI) $(<18.5,18.5-24.9$, $25.0-29.9$ or $\left.\geq 30 \mathrm{~kg} / \mathrm{m}^{2}\right)$, gravidity ( 0 or $\geq 1$ ), parity $(0$ or $\geq 1$ ), infertility duration $(<3,3-6$ or $>6$ years), infertility diagnosis (male factor, tubal factor, other, unexplained or combined), COS protocol (GnRH antagonist, GnRH agonist short, mild stimulation or PPOS), fertilization method (IVF, ICSI or IVF+ICSI), EMT on trigger day $(<8,8-13.9$ or $\geq 14 \mathrm{~mm})$, spontaneous LH surge during $\mathrm{mNC}$ (yes or no), embryos transfer number (single or double), embryo transfer stage (cleavage or blastocyst), transfer of $\geq 1$ top-quality embryo (yes 
Table 1 Baseline characteristics stratified by the percent change of endometrial thickness.

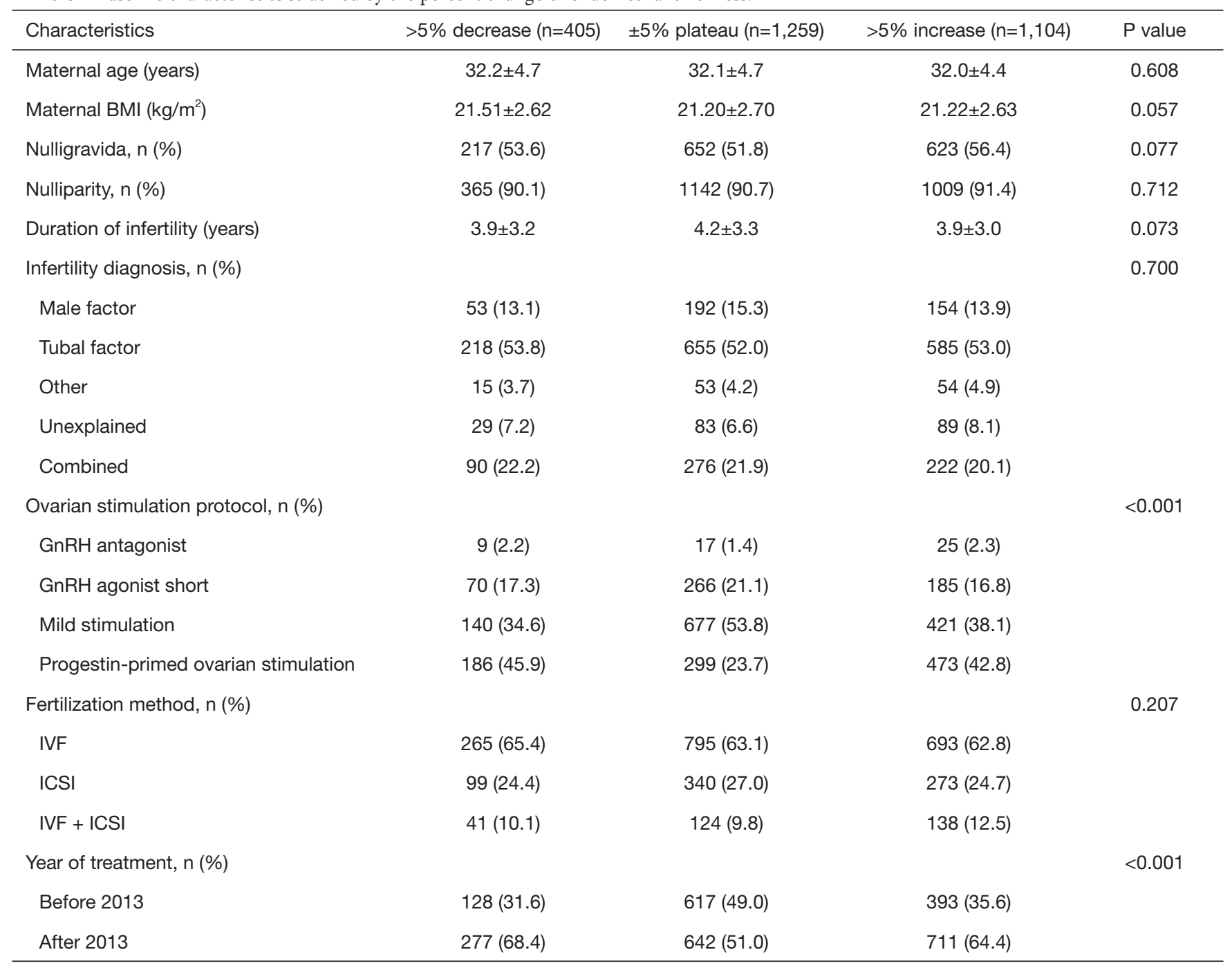

Values are presented as mean \pm standard deviation or number (percentage). BMI, body mass index; GnRH, gonadotropin-releasing hormone; IVF, in vitro fertilization; ICSI, intracytoplasmic sperm injection.

or no) and year of treatment (before or after 2013).

All analyses were conducted with the Statistical Package for the Social Sciences (version 20.0; IBM Corp., USA). Statistical significance was accepted at $\mathrm{P}$ value $<0.05$ on two-sided tests.

\section{Results}

\section{Patient characteristics}

A total of 2768 first mNC-FET cycles were included for analysis (Table 1). The majority of cycles had a plateau $( \pm 5 \%)$ in EMT on FET day compared with that on the day of hCG administration ( $\mathrm{n}=1,259 ; 45.5 \%)$, while the number of cycles with $>5 \%$ post-trigger EMT increase and decrease was 1,104 (39.9\%) and 405 (14.6\%), respectively. Baseline characteristics, including ovarian stimulation protocol and year of treatment, were significantly different among the three groups (both $\mathrm{P}<0.001$ ). No significant differences were found when maternal age, maternal BMI, gravidity, parity, infertility duration, infertility diagnosis and fertilization method were analyzed.

\section{FET cycle characteristics}

Cycle characteristics stratified by the EMT percent change 
Table 2 Cycle characteristics and pregnancy outcomes stratified by the percent change of endometrial thickness

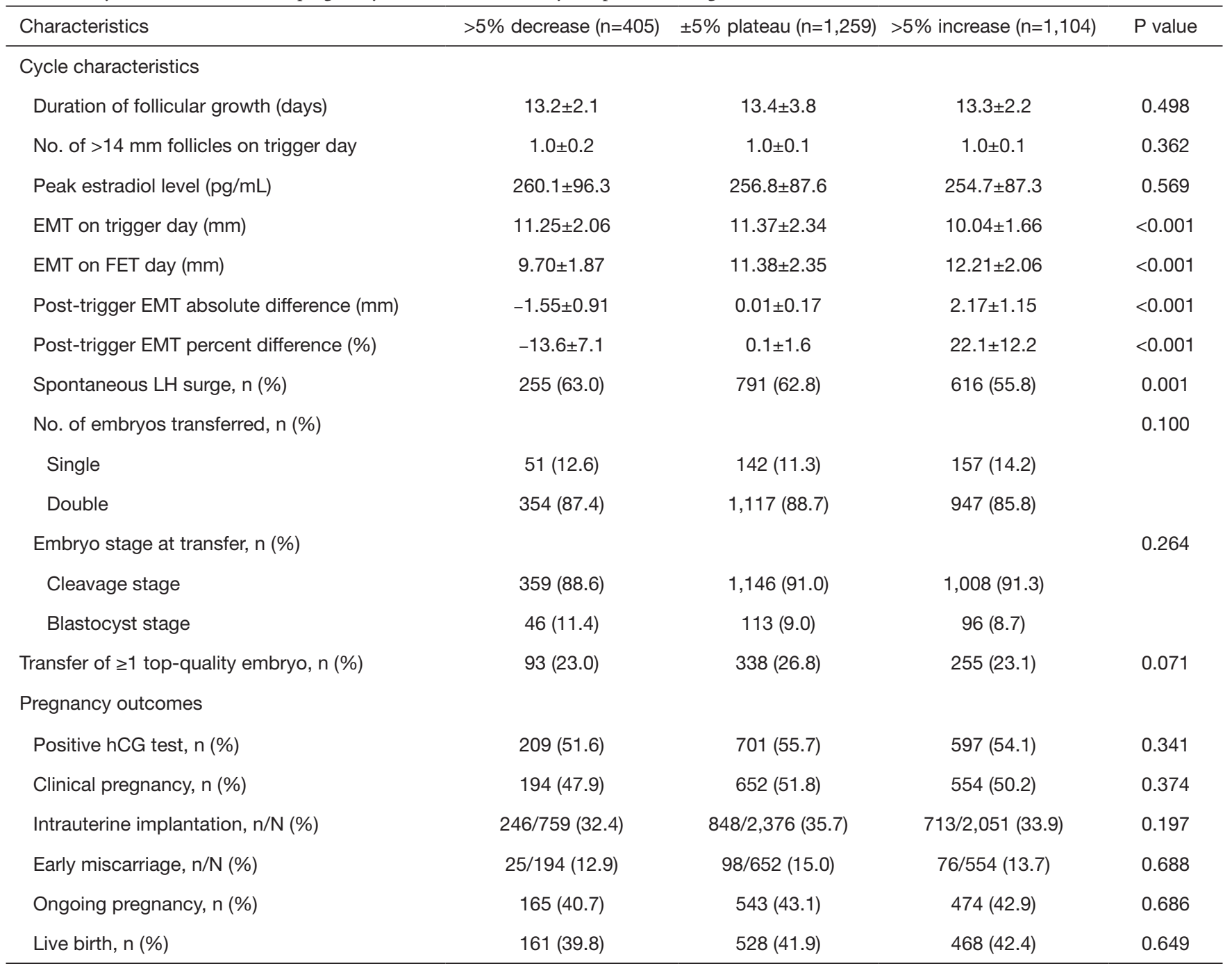

Values are presented as mean \pm standard deviation or number (percentage). EMT, endometrial thickness; FET, frozen-thawed embryo transfer; LH, luteinizing hormone; hCG, human chorionic gonadotrophin.

are presented in Table 2. The mean percent differences of post-trigger EMT were $-13.6 \% \pm 7.1 \%, 0.1 \% \pm 1.6 \%$ and $22.1 \% \pm 12.2 \%$ in the EMT decrease, plateau and increase groups, respectively $(\mathrm{P}<0.001)$. Cycles with EMT $>5 \%$ increase had a significantly lower incidence of spontaneous LH surge compared to those with a plateau or decrease in EMT $(55.8 \%, 62.8 \%$ and $63.0 \%$, respectively; $\mathrm{P}<0.001)$. We found no significant differences with regard to the duration of follicular growth, number of $>14 \mathrm{~mm}$ follicles on trigger day, peak $\mathrm{E}_{2}$ level, embryo transfer number, embryo stage at transfer and the proportion of transferring $\geq 1$ top-quality embryo.

\section{Pregnancy outcomes}

Live birth rates were $41.9 \%, 39.8 \%$ [crude odds ratio (cOR) $0.91,95 \%$ CI, $0.73-1.15]$ and $42.4 \%$ (cOR 1.02, 95\% CI, $0.87-1.20)$ in the EMT $\pm 5 \%$ plateau, $>5 \%$ decrease and $>5 \%$ increase groups, respectively $(\mathrm{P}=0.649)$ (Table 2; Figure 1). Multiple regression analysis did not alter the finding after controlling for a variety of confounding factors. Compared with the post-trigger EMT plateau group, the adjusted OR of live birth was 0.88 (95\% CI, 0.69-1.12) in the decrease group and 1.05 (95\% CI, 0.88-1.25) in the increase group (Figure 1). Similarly, no significant associations were 
A

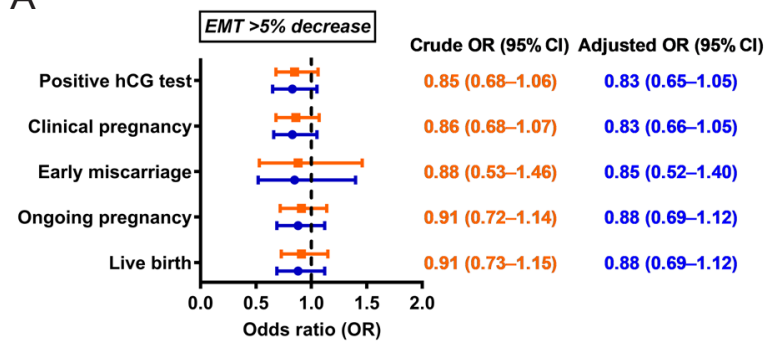

B

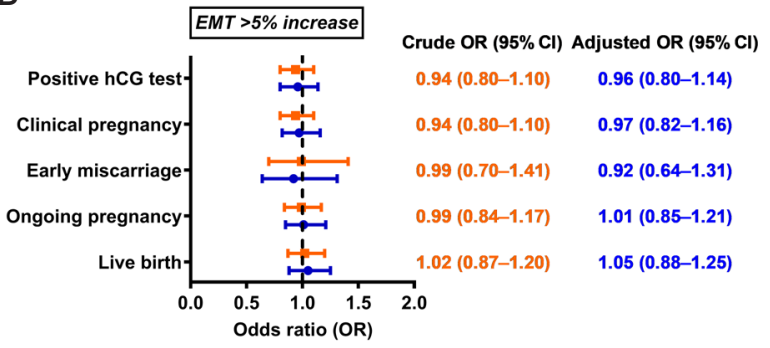

Figure 1 Crude and adjusted odds ratios of pregnancy outcomes for (A) EMT >5\% decrease and (B) EMT >5\% increase in comparison with the EMT plateau $( \pm 5 \%)$ group. EMT, endometrial thickness

observed before or after adjustment between EMT change and other pregnancy outcomes including positive hCG test, clinical pregnancy, early miscarriage and ongoing pregnancy.

\section{Subgroup analyses}

Table 3 demonstrates the results of subgroup analyses for different embryo transfer numbers, embryo developmental stages and EMT categories on trigger day. Throughout the three subanalyses, we detected no statistical significances in both crude and adjusted ORs of live birth for either the decreased or increased EMT group compared with the plateau group. Considering the difference of EMT change between cycles with and without spontaneous LH surge (Figure S1B,C), subgroup analysis was also performed based on this characteristic and the non-significant relationship with live birth was maintained.

\section{Discussion}

In this large retrospective cohort study, we showed for the first time that the change of endometrial lining thickness between the day of hCG triggering and the day of embryo transfer was not associated with pregnancy outcomes in mNC-FET cycles.

In correlation with the circulating levels of ovarian steroid hormones, the endometrium undergoes histological changes periodically during the menstrual cycle (22). In the follicular phase, the $\mathrm{E}_{2}$ exposure promotes the proliferation of luminal and glandular epithelium and stroma. After ovulation, the luteal phase endometrium is then featured by $\mathrm{P}$-induced secretory differentiation of the glands, vascularization of the stroma and pre-decidualization. Correspondingly, with the use of various imaging techniques such as transabdominal ultrasonography, two- or three- dimensional TVU and magnetic resonance imaging, EMT has been reported in most studies to increase during the preovulatory phase, reach the peak in the peri-ovulatory period, and remain unchanged or slightly rise during the postovulatory phase (13-21). In the present study focusing on $\mathrm{mNC}$ protocol, we observed a general increase of EMT (around 7\%) after hCG triggering for ovulation in the whole population, with over $85 \%$ of cycles exhibiting an EMT increase or plateau. This trend coincides well with the EMT change from late follicular to luteal phase displayed in natural menstrual cycles.

A thin endometrium has been well-established to negatively affect the chance of achieving a pregnancy in FET cycles (11). In these observational studies, peak EMT was recorded solely on a single time-point such as the $\mathrm{P}$ starting day or the day of embryo transfer. To date, only three studies have tried to explore the potential relationship between follicular-to-luteal EMT change and pregnancy outcomes (23-25). In 2009, Barker et al. (23) first reported that there was no significant difference of EMT change in pregnant $v s$. non-pregnant recipients $(+1.3 v s .+0.7 \mathrm{~mm})$ after analyzing 79 fresh oocyte donation cycles with blastocyst transfer. However, this study may not have adequate statistical power given the small sample size. Subsequently, Colombo et al. (24) performed a retrospective study of 1313 first FET cycles for women below 35 years with the use of autologous oocytes and transfer of euploid blastocysts. The ongoing pregnancy rates were similar between cycles stratified by EMT decrease $\geq 5 \mathrm{~mm}$ $(\mathrm{n}=310)$ and $<5 \mathrm{~mm}(\mathrm{n}=1,003)$ from $\mathrm{P}$ initiation to the day prior to transfer $(72.2 \%$ vs. $74.5 \%)$, and remained nonsignificant even after controlling for maternal age, BMI and acupuncture use (adjusted OR 0.95, 95\% CI, 0.71-1.28). Nonetheless, no information was provided regarding 
Table 3 Subgroup analysis of live birth rate for different treatment characteristics

\begin{tabular}{|c|c|c|c|}
\hline Characteristics & $>5 \%$ decrease & $\pm 5 \%$ plateau & $>5 \%$ increase \\
\hline \multicolumn{4}{|l|}{ Single } \\
\hline Live birth, n/N (\%) & $16 / 51(31.4)$ & $34 / 142(23.9)$ & 47/157 (29.9) \\
\hline Crude OR (95\% Cl) & $1.45(0.72-2.94)$ & Reference & $1.36(0.81-2.27)$ \\
\hline Adjusted OR (95\% Cl) & $1.65(0.74-3.68)$ & Reference & $1.59(0.89-2.86)$ \\
\hline Live birth, n/N (\%) & $145 / 354(41.0)$ & $494 / 1,117(44.2)$ & $421 / 947(44.5)$ \\
\hline Crude OR (95\% Cl) & $0.88(0.69-1.12)$ & Reference & $1.01(0.85-1.20)$ \\
\hline Adjusted OR (95\% Cl) & $0.84(0.65-1.08)$ & Reference & $1.01(0.84-1.22)$ \\
\hline \multicolumn{4}{|l|}{ Embryo developmental stage } \\
\hline Adjusted OR (95\% Cl) & $0.81(0.62-1.04)$ & Reference & $1.06(0.88-1.27)$ \\
\hline \multicolumn{4}{|l|}{ Blastocyst stage } \\
\hline Live birth, n/N (\%) & $27 / 46(58.7)$ & $51 / 113(45.1)$ & $41 / 96(42.7)$ \\
\hline Crude OR $(95 \% \mathrm{Cl})$ & $1.73(0.86-3.46)$ & Reference & $0.91(0.52-1.57)$ \\
\hline Adjusted OR (95\% Cl) & $1.70(0.76-3.82)$ & Reference & $0.84(0.44-1.61)$ \\
\hline \multicolumn{4}{|c|}{ Endometrial thickness on trigger day } \\
\hline \multicolumn{4}{|l|}{$<8 \mathrm{~mm}$} \\
\hline Live birth, n/N (\%) & $4 / 16(25.0)$ & $14 / 41(34.1)$ & $24 / 80(30.0)$ \\
\hline \multicolumn{4}{|l|}{$\geq 8 \mathrm{~mm}$} \\
\hline Adjusted OR (95\% Cl) & $0.88(0.69-1.13)$ & Reference & $1.04(0.87-1.24)$ \\
\hline \multicolumn{4}{|l|}{$\geq 14 \mathrm{~mm}$} \\
\hline Live birth, n/N (\%) & 20/42 (47.6) & $81 / 162(50.0)$ & 9/22 (40.9) \\
\hline Crude OR (95\% Cl) & $0.91(0.46-1.79)$ & Reference & $0.69(0.28-1.71)$ \\
\hline Adjusted OR (95\% Cl) & $0.73(0.33-1.58)$ & Reference & $0.87(0.32-2.38)$ \\
\hline \multicolumn{4}{|l|}{ Spontaneous LH surge } \\
\hline \multicolumn{4}{|l|}{ No } \\
\hline Live birth, n/N (\%) & $62 / 150(41.3)$ & 199/468 (42.5) & 210/488 (43.0) \\
\hline Crude OR (95\% Cl) & $0.95(0.66-1.38)$ & Reference & $1.02(0.79-1.32)$ \\
\hline Adjusted OR (95\% Cl) & $0.91(0.61-1.36)$ & Reference & $1.07(0.80-1.41)$ \\
\hline \multicolumn{4}{|l|}{ Yes } \\
\hline Live birth, n/N (\%) & 99/255 (38.8) & $329 / 791$ (41.6) & 258/616 (41.9) \\
\hline Crude OR (95\% Cl) & $0.89(0.67-1.19)$ & Reference & $1.01(0.82-1.25)$ \\
\hline Adjusted OR (95\% Cl) & $0.85(0.63-1.16)$ & Reference & $1.04(0.83-1.31)$ \\
\hline
\end{tabular}

OR, odds ratio; $\mathrm{Cl}$, confidence interval. 
FET treatment characteristics such as the duration of $\mathrm{E}_{2}$ administration, whose prolongation has been shown to be associated with reduced pregnancy rates (31). On the contrary, a recent study by Haas et al. (25) from Toronto reviewed 271 single blastocyst FET cycles and found a highly significant inverse relationship between EMT change and ongoing pregnancy rate. This report, together with the other two studies, were all conducted in artificial cycles only, failed to discriminate groups of EMT plateau with increase and lacked the key outcome of live birth rate.

The finding of our study agrees with the results of Barker et al. (23) and Colombo et al. (24), but contradicts the work of Haas et al. (25). This discrepancy may be explained by the differences in study design and implementation. As stated by the authors, endometrial measurement was performed by TVU at the end of the follicular/estrogen phase, but was replaced with transabdominal ultrasound on the day of FET. Instead of gathering data in a prospective manner, EMT was taken after retrospectively retrieving and analyzing ultrasound images with specific software. Consequently, they observed a rather high incidence $(42.4 \%)$ of EMT compaction $\geq 5 \%$ in response to $\mathrm{P}$ administration, which is discordant with the normal follicular-to-luteal EMT change during the menstrual cycle (13-21), artificial FET cycle $(23,24)$ as well as $\mathrm{mNC}$ in our study. In addition, hormone therapy plus GnRH agonist suppression or not was combined as a whole by Haas et al. (25) without further adjustment. This, therefore, might have led to a biased interpretation of the results, given the significant differences in clinical pregnancy and live birth rates between the two treatments (32).

According to data from multivariable regression analysis (Table S1), the reduced likelihood of live birth was associated with older age, higher BMI, longer infertility duration and some specific etiologies of infertility. In contrast, factors that positively correlated with FET success included double, blastocyst-stage and top-quality embryo transfers. These clinical parameters were well in consistency with existing literatures (33-35). The odds of live birth decreased in patients with thin endometrial linings (EMT $<8 \mathrm{~mm}$ ), yet the difference failed to reach statistical significance (adjusted OR 0.69, 95\% CI, 0.47-1.01). Similar to the previous finding from a prospective trial (36), spontaneous LH surges occurred in over half of cycles prior to hCG administration, but demonstrated no adverse influence on the likelihood of pregnancy in mNC-FET protocol.

A major strength of our study lies in the large sample size of $>2,700$ first FET cycles within a single institution. The complete electronic records with prospectively collected data allow us to exclude patients with congenital and acquired uterine anomalies that could potentially interfere with the accuracy of EMT measurement and adversely affect pregnancy outcomes. It also enables our conduction of multiple regression and subgroup analyses to control for various confounders, thus improving the validity and robustness of our results.

Despite our caution, there are still certain shortcomings to be acknowledged. Firstly, our study was retrospective in design and other unknown confounding factors may not be accounted for. Significant differences were found in some baseline characteristics including COS protocols and year of treatment. Nonetheless, these parameters had no effects on live birth outcome as indicated in the multivariable regression model. Secondly, regular ovulatory women undergoing $\mathrm{mNC}$ preparation were intentionally screened to facilitate our evaluation of follicular-to-luteal EMT change mimicking the natural menstrual cycle. Therefore, the generalization of our study conclusion should not be directly extrapolated to fresh IVF cycles or FET cycles using hormonal therapy and ovulation induction. Thirdly, double cleavage-stage embryo transfer was the principal strategy in our and most other centers in China during the study period $(37,38)$. While subgroup analyses according to embryo number and developmental stage did not alter the conclusion, future studies focusing on single euploid blastocyst transfer cycles would be necessary for further confirmation. Finally, although EMT measurement was conducted by highly trained sonographers in our center, the presence of intra- and inter-observer inconsistency remains to be an insurmountable limitation. In addition, endometrial pattern, volume and vascularity have also been shown to fluctuate during the menstrual cycle $(39,40)$, but these indexes were not assessed and accordingly, the finding of this work is restricted to the EMT change only.

\section{Conclusions}

In summary, our study suggests no association between EMT change from hCG administration to embryo transfer and pregnancy chances in mNC-FET cycles. Given concerns raised by patients regarding their EMT decrease on FET day, our results would provide reassuring information for counseling. From our point of view, remeasurement of EMT on the day of transfer does not appear to add significant clinical value and thus should not 
be routinely recommended in current practice. Since EMT exhibits variations at different cycle time-points among different patients, the present study also partially explains why a single EMT assessment for endometrial receptivity offers only a poor predictive ability for FET treatment outcomes.

\section{Acknowledgments}

The authors would like to express sincere gratitude to Dr. Sicheng Wu, Clinical Research Center of Shanghai Ninth People's Hospital affiliated to Shanghai Jiao Tong University School of Medicine, for his support in data management and statistical analysis.

Funding: This work was supported by the National Key Research and Development Program of China (Grant No. 2018YFC1003000), National Natural Science Foundation of China (Grant No. 81771533), Elite Group Project of Shanghai Ninth People's Hospital (Grant No. JY201801) and Latitudinal Research Project of Shanghai Jiao Tong University School of Medicine (Grant No. 2017hx005/ DK3-0601-17-0007).

\section{Footnote}

Reporting Checklist: The authors have completed the MDAR checklist and STROBE reporting checklist. Available at http://dx.doi.org/10.21037/atm-20-1459

Data Sharing Statement: Available at http://dx.doi. org/10.21037/atm-20-1459

Conflicts of Interest: All authors have completed the ICMJE uniform disclosure form (available at http://dx.doi. org/10.21037/atm-20-1459). The authors have no conflicts of interest to declare.

Ethical Statement: The authors are accountable for all aspects of the work in ensuring that questions related to the accuracy or integrity of any part of the work are appropriately investigated and resolved. The study was conducted in accordance with the Declaration of Helsinki (as revised in 2013). The study was approved by the institutional review board of Shanghai Ninth People's Hospital affiliated with Shanghai Jiao Tong University School of Medicine (No. 2017-211) and individual consent for this retrospective analysis was waived.
Open Access Statement: This is an Open Access article distributed in accordance with the Creative Commons Attribution-NonCommercial-NoDerivs 4.0 International License (CC BY-NC-ND 4.0), which permits the noncommercial replication and distribution of the article with the strict proviso that no changes or edits are made and the original work is properly cited (including links to both the formal publication through the relevant DOI and the license). See: https://creativecommons.org/licenses/by-nc-nd/4.0/.

\section{References}

1. Trounson A, Mohr L. Human pregnancy following cryopreservation, thawing and transfer of an eight-cell embryo. Nature 1983;305:707-9.

2. Doody KJ. Cryopreservation and delayed embryo transferassisted reproductive technology registry and reporting implications. Fertil Steril 2014;102:27-31.

3. De Geyter C, Calhaz-Jorge C, Kupka MS, et al. ART in Europe, 2014: results generated from European registries by ESHRE: The European IVF-monitoring Consortium (EIM) for the European Society of Human Reproduction and Embryology (ESHRE). Hum Reprod 2018;33:1586-601.

4. Wong KM, van Wely M, Mol F, et al. Fresh versus frozen embryo transfers in assisted reproduction. Cochrane Database Syst Rev 2017;3:CD011184.

5. Roque M, Haahr T, Geber S, et al. Fresh versus elective frozen embryo transfer in IVF/ICSI cycles: a systematic review and meta-analysis of reproductive outcomes. Hum Reprod Update 2019;25:2-14.

6. Sha T, Yin X, Cheng W, et al. Pregnancy-related complications and perinatal outcomes resulting from transfer of cryopreserved versus fresh embryos in vitro fertilization: a meta-analysis. Fertil Steril 2018;109:330-42.e9.

7. Alviggi C, Conforti A, Carbone IF, et al. Influence of cryopreservation on perinatal outcome after blastocystvs cleavage-stage embryo transfer: systematic review and meta-analysis. Ultrasound Obstet Gynecol 2018;51:54-63.

8. Ghobara T, Gelbaya TA, Ayeleke RO. Cycle regimens for frozen-thawed embryo transfer. Cochrane Database Syst Rev 2017;7:CD003414.

9. Mackens S, Santos-Ribeiro S, van de Vijver A, et al. Frozen embryo transfer: a review on the optimal endometrial preparation and timing. Hum Reprod 2017;32:2234-42.

10. Kasius A, Smit JG, Torrance HL, et al. Endometrial thickness and pregnancy rates after IVF: a systematic review and meta-analysis. Hum Reprod Update 
2014;20:530-41.

11. Craciunas L, Gallos I, Chu J, et al. Conventional and modern markers of endometrial receptivity: a systematic review and meta-analysis. Hum Reprod Update 2019;25:202-23.

12. Groenewoud ER, Cohlen BJ, Al-Oraiby A, et al. Influence of endometrial thickness on pregnancy rates in modified natural cycle frozen-thawed embryo transfer. Acta Obstet Gynecol Scand 2018;97:808-15.

13. Haynor DR, Mack LA, Soules MR, et al. Changing appearance of the normal uterus during the menstrual cycle: MR studies. Radiology 1986;161:459-62.

14. Wiczyk HP, Janus CL, Richards CJ, et al. Comparison of magnetic resonance imaging and ultrasound in evaluating follicular and endometrial development throughout the normal cycle. Fertil Steril 1988;49:969-72.

15. Randall JM, Fisk NM, Mc'Tavish A, et al. Transvaginal ultrasonic assessment of endometrial growth in spontaneous and hyperstimulated menstrual cycles. Br J Obstet Gynaecol 1989;96:954-9.

16. Lenz S, Lindenberg S. Ultrasonic evaluation of endometrial growth in women with normal cycles during spontaneous and stimulated cycles. Hum Reprod 1990;5:377-81.

17. Fleischer AC, Gordon AN, Entman SS, et al. Transvaginal scanning of the endometrium. J Clin Ultrasound 1990;18:337-49.

18. Bartoli JM, Moulin G, Delannoy L, et al. The normal uterus on magnetic resonance imaging and variations associated with the hormonal state. Surg Radiol Anat 1991;13:213-20.

19. Santolaya-Forgas J. Physiology of the menstrual cycle by ultrasonography. J Ultrasound Med 1992;11:139-42.

20. Bakos O, Lundkvist O, Bergh T. Transvaginal sonographic evaluation of endometrial growth and texture in spontaneous ovulatory cycles--a descriptive study. Hum Reprod 1993;8:799-806.

21. Hoad CL, Raine-Fenning NJ, Fulford J, et al. Uterine tissue development in healthy women during the normal menstrual cycle and investigations with magnetic resonance imaging. Am J Obstet Gynecol 2005;192:648-54.

22. Noyes RW, Hertig AT, Rock J. Dating the endometrial biopsy. Am J Obstet Gynecol 1975;122:262-3.

23. Barker MA, Boehnlein LM, Kovacs P, et al. Follicular and luteal phase endometrial thickness and echogenic pattern and pregnancy outcome in oocyte donation cycles. J Assist Reprod Genet 2009;26:243-9.

24. Colombo S, Franasiak J, Kaser DJ, et al. Significance of endometrial lining decrease after progesterone start in synthetic frozen embryo transfer (FET) cycles. Fertil Steril 2017;108:e304-5.

25. Haas J, Smith R, Zilberberg E, et al. Endometrial compaction (decreased thickness) in response to progesterone results in optimal pregnancy outcome in frozen-thawed embryo transfers. Fertil Steril 2019;112:503-9.e1.

26. Kuang Y, Chen Q, Fu Y, et al. Medroxyprogesterone acetate is an effective oral alternative for preventing premature luteinizing hormone surges in women undergoing controlled ovarian hyperstimulation for in vitro fertilization. Fertil Steril 2015;104:62-70.e3.

27. Chen H, Wang Y, Lyu Q, et al. Comparison of livebirth defects after luteal-phase ovarian stimulation vs. conventional ovarian stimulation for in vitro fertilization and vitrified embryo transfer cycles. Fertil Steril 2015;103:1194-201.e2.

28. Wang Y, Kuang Y, Chen Q, et al. Gonadotropin-releasing hormone antagonist versus progestin for the prevention of premature luteinising hormone surges in poor responders undergoing in vitro fertilisation treatment: study protocol for a randomised controlled trial. Trials 2018;19:455.

29. Cummins JM, Breen TM, Harrison KL, et al. A formula for scoring human embryo growth rates in in vitro fertilization: its value in predicting pregnancy and in comparison with visual estimates of embryo quality. J In Vitro Fert Embryo Transf 1986;3:284-95.

30. Gardner DK, Schoolcraft WB. In vitro culture of human blastocyst. In: Jansen R, Mortimer D. editors. Towards reproductive certainty: infertility and genetics beyond 1999. Carnforth: Parthenon Press, 1999:378-88.

31. Bourdon M, Santulli P, Kefelian F, et al. Prolonged estrogen (E2) treatment prior to frozen-blastocyst transfer decreases the live birth rate. Hum Reprod 2018;33:905-13.

32. El-Toukhy T, Taylor A, Khalaf Y, et al. Pituitary suppression in ultrasound-monitored frozen embryo replacement cycles. A randomised study. Hum Reprod 2004;19:874-9.

33. Veleva Z, Orava M, Nuojua-Huttunen S, et al. Factors affecting the outcome of frozen-thawed embryo transfer. Hum Reprod 2013;28:2425-31.

34. Dhillon RK, McLernon DJ, Smith PP, et al. Predicting the chance of live birth for women undergoing IVF: a novel pretreatment counselling tool. Hum Reprod 2016;31:84-92.

35. Zhu Q, Zhu J, Wang Y, et al. Live birth rate and neonatal outcome following cleavage-stage embryo transfer versus blastocyst transfer using the freeze-all strategy. Reprod 
Biomed Online 2019;38:892-900.

36. Groenewoud ER, Kollen BJ, Macklon NS, et al. Spontaneous LH surges prior to HCG administration in unstimulated-cycle frozen-thawed embryo transfer do not influence pregnancy rates. Reprod Biomed Online 2012;24:191-6.

37. Chen ZJ, Shi Y, Sun Y, et al. Fresh versus Frozen Embryos for Infertility in the Polycystic Ovary Syndrome. N Engl J Med 2016;375:523-33.

38. Shi Y, Sun Y, Hao C, et al. Transfer of Fresh versus

Cite this article as: Huang J, Lin J, Cai R, Lu X, Song N, Gao H, Kuang Y. Significance of endometrial thickness change after human chorionic gonadotrophin triggering in modified natural cycles for frozen-thawed embryo transfer. Ann Transl Med 2020;8(23):1590. doi: 10.21037/atm-20-1459
Frozen Embryos in Ovulatory Women. N Engl J Med 2018;378:126-36.

39. Baerwald AR, Pierson RA. Endometrial development in association with ovarian follicular waves during the menstrual cycle. Ultrasound Obstet Gynecol 2004;24:453-60.

40. Jokubkiene L, Sladkevicius P, Rovas L, et al. Assessment of changes in endometrial and subendometrial volume and vascularity during the normal menstrual cycle using three-dimensional power Doppler ultrasound. Ultrasound Obstet Gynecol 2006;27:672-9. 
A

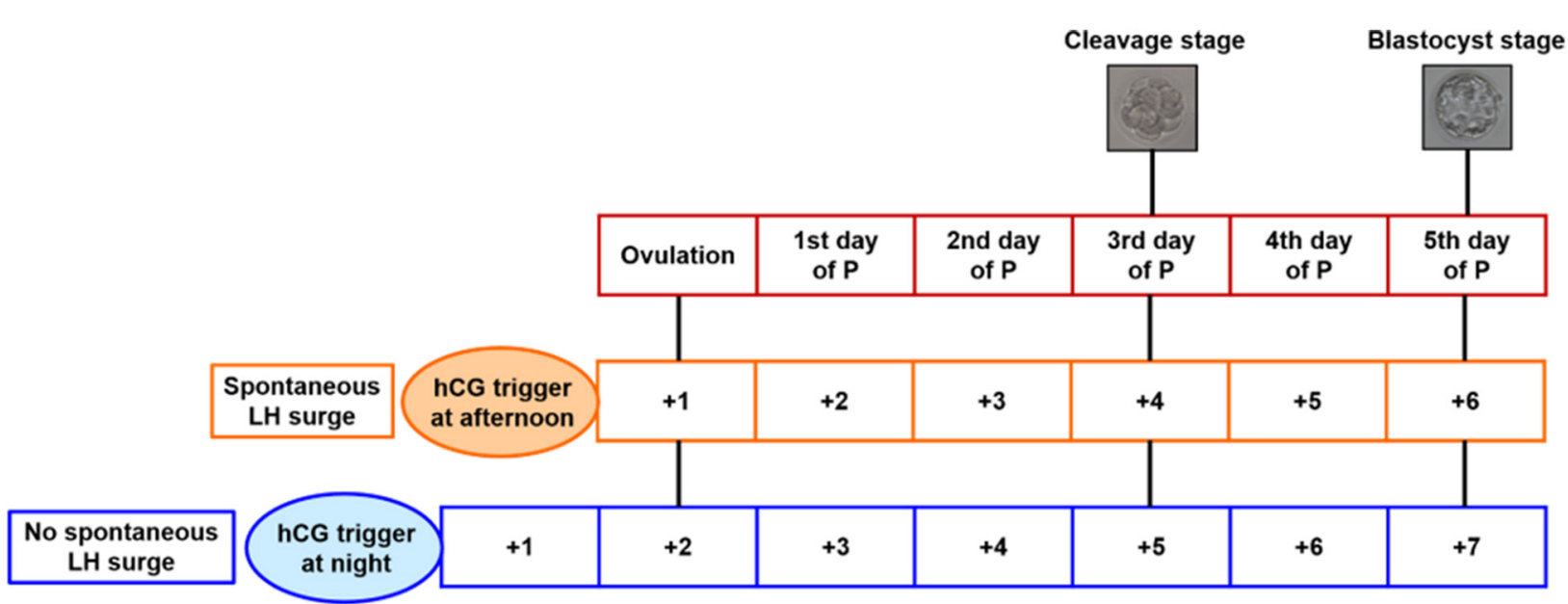

B
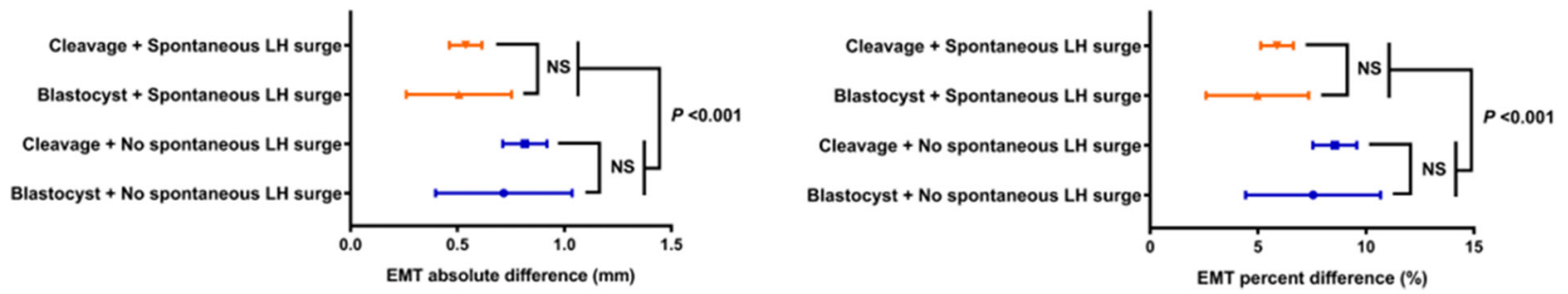

Figure S1 Endometrial thickness (EMT) change after human chorionic gonadotrophin (hCG) triggering in modified natural cycles for frozen-thawed embryo transfer (FET). (A) The scheme of FET timing; (B,C) comparison of absolute and percent changes of post-trigger EMT in cleavage- and blastocyst-stage embryo transfer cycles with or without spontaneous luteinizing hormone (LH) surge. The error bars show the $95 \%$ CIs. P, progesterone; NS, no significance. 


\begin{tabular}{|c|c|c|c|}
\hline & Adjusted OR (95\% Cl) & Wals & P-value \\
\hline \multicolumn{4}{|l|}{ Maternal age (years) } \\
\hline$<30$ & Reference & & \\
\hline $30-34$ & $0.86(0.72-1.04)$ & 2.481 & 0.115 \\
\hline $35-37$ & $0.62(0.47-0.80)$ & 12.734 & $<0.001$ \\
\hline $38-40$ & $0.56(0.39-0.79)$ & 11.095 & 0.001 \\
\hline$\geq 41$ & $0.11(0.06-0.21)$ & 52.252 & $<0.001$ \\
\hline \multicolumn{4}{|l|}{ Maternal BMI (kg/m²) } \\
\hline$<18.5$ & $0.86(0.68-1.10)$ & 1.457 & 0.227 \\
\hline $18.5-24.9$ & Reference & & \\
\hline $25.0-29.9$ & $1.05(0.77-1.44)$ & 0.108 & 0.742 \\
\hline$\geq 30$ & $0.46(0.22-0.99)$ & 3.868 & 0.049 \\
\hline Gravidity ( $\geq 1$ vs. 0 ) & $0.95(0.79-1.13)$ & 0.355 & 0.551 \\
\hline Parity ( $\geq 1$ vs. 0) & $1.08(0.79-1.48)$ & 0.239 & 0.625 \\
\hline \multicolumn{4}{|l|}{ Duration of infertility (years) } \\
\hline$<3$ & Reference & & \\
\hline $3-6$ & $0.98(0.82-1.17)$ & 0.045 & 0.832 \\
\hline$>6$ & $0.77(0.60-0.99)$ & 4.264 & 0.039 \\
\hline \multicolumn{4}{|l|}{ Infertility diagnosis } \\
\hline Male factor & Reference & & \\
\hline Tubal factor & $0.68(0.51-0.91)$ & 6.855 & 0.009 \\
\hline Other & $0.78(0.49-1.23)$ & 1.171 & 0.279 \\
\hline Unexplained & $0.87(0.59-1.28)$ & 0.478 & 0.489 \\
\hline Combined & $0.74(0.56-0.99)$ & 4.208 & 0.040 \\
\hline \multicolumn{4}{|l|}{ Ovarian stimulation protocol } \\
\hline GnRH antagonist & Reference & & \\
\hline GnRH agonist short & $0.68(0.37-1.25)$ & 1.513 & 0.219 \\
\hline Mild stimulation & $0.86(0.47-1.54)$ & 0.272 & 0.602 \\
\hline Progestin-primed ovarian stimulation & $1.05(0.57-1.92)$ & 0.022 & 0.882 \\
\hline \multicolumn{4}{|l|}{ Fertilization method } \\
\hline IVF & Reference & & \\
\hline ICSI & $0.86(0.68-1.09)$ & 1.614 & 0.204 \\
\hline IVF + ICSI & $0.85(0.64-1.12)$ & 1.316 & 0.251 \\
\hline Year of treatment (after vs. before 2013) & $0.97(0.80-1.16)$ & 0.140 & 0.708 \\
\hline \multicolumn{4}{|l|}{ EMT on trigger day (mm) } \\
\hline$<8$ & $0.69(0.47-1.01)$ & 3.565 & 0.059 \\
\hline $8-13.9$ & Reference & & \\
\hline$\geq 14$ & $1.20(0.90-1.61)$ & 1.555 & 0.212 \\
\hline \multicolumn{4}{|l|}{ Post-trigger EMT percent difference } \\
\hline$>5 \%$ decrease & $0.88(0.69-1.12)$ & 1.049 & 0.306 \\
\hline $\pm 5 \%$ plateau & Reference & & \\
\hline$>5 \%$ increase & $1.05(0.88-1.25)$ & 0.244 & 0.621 \\
\hline Spontaneous LH surge (yes vs. no) & $0.96(0.82-1.13)$ & 0.250 & 0.617 \\
\hline No. of embryos transferred (double vs. single) & $2.22(1.69-2.93)$ & 32.494 & $<0.001$ \\
\hline Embryo stage at transfer (blastocyst vs. cleavage) & $1.63(1.22-2.19)$ & 10.607 & 0.001 \\
\hline Transfer of $\geq 1$ top-quality embryo (yes vs. no) & $1.21(1.01-1.46)$ & 4.022 & 0.045 \\
\hline
\end{tabular}

$\mathrm{OR}$, odds ratio; $\mathrm{Cl}$, confidence interval; BMI, body mass index; $\mathrm{GnRH}$, gonadotropin-releasing hormone; IVF, in vitro fertilization; ICSI, intracytoplasmic sperm injection; EMT, endometrial thickness; LH, luteinizing hormone. 